\title{
On Convex Bodies that are Characterizable by Volume Function
}

\section{"Old and Recent Problems for a New Generation": A Survey}

\author{
Ákos G. Horváth ${ }^{1}$
}

Received: 29 July 2019 / Revised: 3 January 2020 / Accepted: 8 January 2020 / Published online: 23 January 2020 (c) The Author(s) 2020

\begin{abstract}
The "old-new" concept of a convex-hull function was investigated by several authors in the last seventy years. A recent research on it led to some other volume functions as the covariogram function, the widthness function or the so-called brightness functions, respectively. A very interesting fact that there are many long-standing open problems connected with these functions whose serious investigation was closed before the "age of computers". In this survey, we concentrate only on the three-dimensional case; we will mention the most important concepts, statements, and problems.
\end{abstract}

Keywords Centred convex body $\cdot$ Central symmetric convex body $\cdot$ Convex-hull function · Covariogram function · Difference body $\cdot$ Blaschke body

Mathematics Subject Classification 52A40 $\cdot 52 \mathrm{~A} 38 \cdot 26 \mathrm{~B} 15 \cdot 52 \mathrm{~B} 11$

\section{Introduction}

The "old-new" concept of a convex-hull function was investigated by several authors in the last seventy years. A recent research on it led to some other volume functions as the covariogram function, the widthness function or the so-called brightness functions, respectively. A very interesting fact is that there are many long-standing open problems connected with these functions whose serious investigation was closed before the "age of computers". The structure of the conjectured optimal bodies reflects quite a theoretical attitude: seemingly, there was no computer research to support them. In this paper, we collect some of them (using the necessary theoretical knowledge) to inspire the computer experts for such research which can reorder the map of these problems. We

Ákos G. Horváth

ghorvath@math.bme.hu

1 Department of Geometry, Mathematical Institute, Budapest University of Technology and Economics, Budapest 1521, Hungary 
concentrate on the three-dimensional case; we mention the most important concepts, statements, and problems.

We use the following notation. $K$ is a convex body; the class of convex bodies is denoted by $\mathcal{K} . K+L$ denotes the Minkowski sum of the convex bodies $K$ and $L$ and $-K$ is the reflected image of $K$ with respect to the origin. $\mathbb{R}^{n}$ and $\mathcal{S}^{n-1}$ are the analytic models of the $n$-dimensional Euclidean space and the $(n-1)$-dimensional sphere, respectively. A convex body with continuously differentiable boundary is called a $C^{1}$ body; analogously, a convex body with boundary of positive Gauss curvature is called a $C_{+}^{2}$-body. We use the following special notation:

- $D K$ : the difference body of $K$

- $\triangle K$ : the central symmetral of $K$

- $\Pi K$ : the projection body of $K$

- $\nabla K$ : the Blaschke body of $K$

- $g_{K}(u)$ : the covariogram function of $K$

- $h_{K}(u)$ : the support function of $K$

- $w_{K}(u)$ : the width function of $K$

- $b_{K}(u)$ : the brightness function of $K$

- $G_{K}(u)$ : the convex-hull function of $K$

- $G(u)$ : the Gauss map.

From the general theory of convex sets, we use the concept of Minkowski norm generated by a convex body centrally symmetric with respect to the origin; the concept of polar body and the special 2-dimensional norm, the so-called Radon norm with the boundary of its unit disk being a Radon curve. The following statements are important in our arguments:

- Brunn-Minkowski's inequality: If $K$ and $L$ are convex bodies then

$$
\operatorname{vol}_{n}(K+L)^{\frac{1}{n}} \leq \operatorname{vol}_{n}(K)^{\frac{1}{n}}+\operatorname{vol}_{n}(L)^{\frac{1}{n}}
$$

- Alexandrov's projection theorem: Let $1 \leq i \leq k \leq n-1$, and let $K, L$ be centrally symmetric compact convex sets of dimension at least $i+1$ in $\mathbb{R}^{n}$. If $V_{i}(K \mid S)=V_{i}(L \mid S)$ for all $k$-dimensional subspaces $S$ of $\mathbb{R}^{n}$, then $K$ is a translate of $L$.

- Cauchy's projection formula:

$$
V\left(K \mid u^{\perp}\right)=\frac{1}{2} \int_{\mathcal{S}^{n-1}}\langle u, u\rangle \mathrm{d} S(K, v) .
$$

- Minkowski's existence theorem: For the finite Borel measure $\mu$ in $\mathcal{S}^{n-1}$ to be $S_{n-1}(K, \cdot)$ for some convex body $K \in \mathcal{K}$, it is necessary and sufficient that $\mu$ is not concentrated on any great subsphere of $\mathcal{S}^{n-1}$, and

$$
\int_{\mathcal{S}^{n-1}} u \mathrm{~d} \mu(u)=0 .
$$


- Minkowski's first inequality: Let $V^{n}(K, n-1 ; L)$ be the mixed volume $V^{n}(K, \ldots, K, L)$ where the number of the copies of $K$ is $n-1$; then

$$
V^{n}(K, n-1 ; L) \geq \operatorname{vol}_{n}^{n-1}(K) \operatorname{vol}_{n}(L),
$$

with equality if and only if $K$ and $L$ lie in parallel hyperplanes or are homothetic.

\section{The Covariogram Function}

Let $K$ be a convex body in $\mathbb{R}^{n}$. The covariogram $g_{K}$ of $K$ is the function

$$
g_{K}(t):=\operatorname{vol}_{n}(K \cap(K+t))
$$

where $t \in \mathbb{R}^{n}$ and $\operatorname{vol}_{n}$ denotes $n$-dimensional volume. This functional, which was introduced by Matheron in his book Matheron (1986) on random sets, is also sometimes called the set covariance and it coincides with the autocorrelation of the characteristic function $1_{K}$ of $K$ :

$$
g_{K}=\mathbf{1}_{K} \star \mathbf{1}_{-K}
$$

The covariogram $g_{K}$ is clearly unchanged by a translation or a reflection of $K$. (The term reflection will always mean reflection in a point.) Matheron (1986) asked the following question and conjectured a positive answer for the case $n=2$.

Problem 1 (Covariogram problem) Does the covariogram determine a convex body among all convex bodies up to translations and reflections?

The first contribution to Matheron's question was made by Nagel (1993), who confirmed Matheron's conjecture for all convex polygons. Other partial results towards the complete confirmation of this conjecture in the plane have been proved by Schmitt (1993), Bianchi et al. (2002), Bianchi (2005), Averkov and Bianchi (2007). In general, the answer to the covariogram problem is negative, as the author of Bianchi (2005) proved by finding counterexamples in $\mathbb{R}^{n}$ for any $n \geq 4$. Indeed, the covariogram of the Cartesian product of convex sets $K \subset \mathbb{R}^{k}$ and $L \subset \mathbb{R}^{m}$ is the product of the covariograms of $K$ and $L$. Thus $K \times L$ and $K \times(-L)$ have equal covariograms. However, if neither $K$ nor $L$ is centrally symmetric, then $K \times L$ is neither a translation nor a reflection of $K \times(-L)$. To satisfy these requirements, the dimensions of both sets must be at least two and thus the dimension of the counterexamples must be at least four. We note that these counterexamples can be polytopes but not $C^{1}$ bodies. For $n$-dimensional convex polytopes $P$, Goodey et al. (1997) proved that if $P$ is simplicial and $P$ and $-P$ are in general relative position (the polytope is a generic polytope), the covariogram determines $P$. Finally, Bianchi (2009) proved that for three-dimensional polytopes the conjecture is also true. We note that the general case of dimension three is open: it is still not known whether every three-dimensional convex body is determined by its covariogram. We collected the most important statements in the following theorem: 

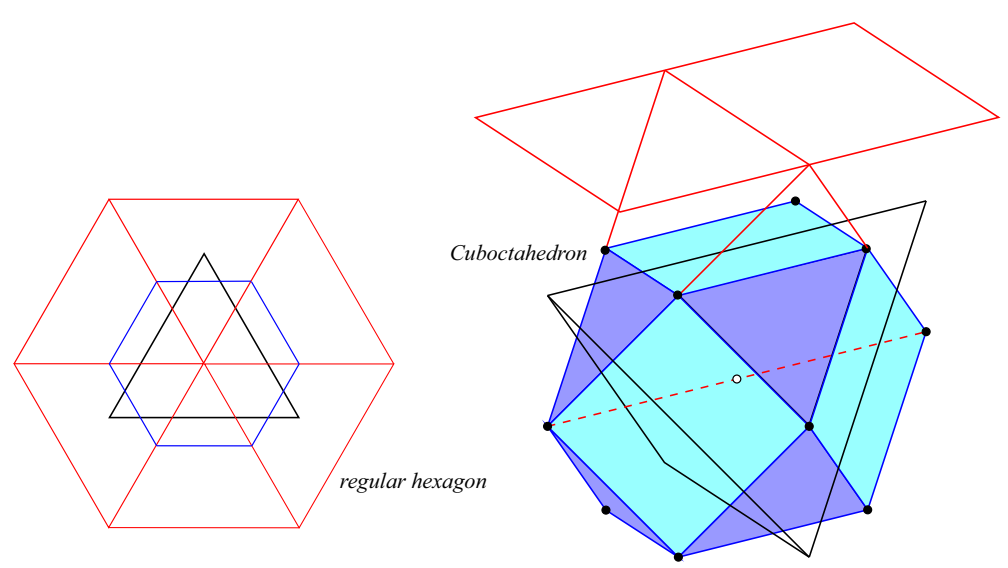

Fig. 1 The difference bodies of simplices

Theorem 1 Let $K$ be a convex body of dimension $n$ in the Euclidean $n$-space.

- The covariogram function determines a convex body $K$ among all convex bodies up to translations and reflections in the following cases:

- $K$ is centrally symmetric,

- $\operatorname{dim} K=2$,

- $K$ is a polytope, and $\operatorname{dim} K=3$,

- $K$ is a generic polytope of dimension $n$.

- If $n \geq 4$, there are convex polytopes which are not determined by their covariogram function.

We first deal with such bodies which are related to this nice problem.

\subsection{The Difference Body and the Central Symmetral of $K$}

First observe that the covariogram function $g_{K}$ determines the volume of $K$, since $g_{K}(0)=\operatorname{vol}_{n}(K)$. What the covariogram obviously does determine is its support, which is the convex body

$$
\mathrm{D} K:=\left\{x \in \mathbb{R}^{n}: K \cap(K+x) \neq \emptyset\right\}=K-K
$$

This is the difference body of $K$. Sometimes is more convenient to define the socalled central symmetral $\triangle K$ of $K$ which is the homothetic by factor $1 / 2$ copy of the difference body. The difference body can be constructed as follows. Suppose that the origin $o$ is in the interior of $K$, and take the union of all translates of $-K$ which are placed so that the corresponding translate of $o$ lies on the boundary of $K$. Finally, if we dilate by a factor $1 / 2$, we get $\Delta K$. The central symmetral of a regular triangle is a regular hexagon, and the central symmetral of a regular tetrahedron is the cuboctahedron (see Fig. 1). Since for a centrally symmetric convex body $K$ with 
center $c$, the equality $-K=K-2 c$ holds,

$$
t K+(1-t)(-K)=K-2 c(1-t),
$$

and in particular $\Delta K$ is a translate of $K$. Hence a centrally symmetric convex body is determined (among all convex bodies) by its covariogram, since $2 K$ is a translated copy of $\mathrm{D} K$. In general, $\triangle K$ does not determine $K$ meaning that there are non-congruent convex bodies with the same central symmetral.

\subsection{The Support Function and the Width Function}

The support function $h_{K}$ of $K$ is defined by

$$
h_{K}(x)=\max \{\langle x, y\rangle: y \in K\}
$$

for $x \in \mathbb{R}^{n}$. As a function, the support function is positively homogeneous and subadditive, that is, a sublinear function. It can be observed that a convex body is determined by its support function [see (0.6) in Gardner (1995)].

Let $K$ be a compact, convex set in $\mathbb{R}^{n}$. Then $K$ has two supporting hyperplanes, which are orthogonal to a unit vector $u$. The distance between these hyperplanes is the width $w_{K}(u)$ of $K$ in the direction of $u$. The width of $K$ is the maximal value of the width function; the thickness of $K$ is the minimal one, respectively. The formal definition of the width function is

$$
w_{K}(u):=h_{K}(u)+h_{K}(-u)
$$

for $u \in \mathcal{S}^{n-1}$. Since

$$
w_{t K+(1-t) K}=t w_{K}+(1-t) w_{-K}=w_{K},
$$

there is a whole continuum of non-congruent compact convex sets, which have the same width function. One of them is the central symmetral of $K$, which shows that $w_{\triangle K}=w_{K}$ for all convex compact bodies. (Generally, $\triangle K$ is the unique centered compact convex set with this property.)

The support function of the central symmetral is equal to $h_{\triangle K}=1 / 2\left(h_{K}+h_{-K}\right)=$ $1 / 2 w_{K}$; thus the support function of the difference body is the width function of $\mathrm{K}$. In particular, the covariogram of a convex body determines its width function, but this property also allows a great uncertainness in the determination of $K$. We can define the class of convex bodies with constant width by the equation

$$
w_{K}(u)=h_{K}(u)+h_{K}(-u)=\text { constant. }
$$

Clearly, the $n$-dimensional ball is of constant width. On the plane, nonspherical examples are the Reuleaux polygons, and there is an analogous theorem in any dimension. Precisely, it can be proved that nonspherical convex bodies of constant width exist 
in $\mathbb{R}^{n}$ for all $n \geq 2$ [see Theorem 3.2.5 in Gardner (1995)]. There are nice investigations of this class of bodies. Evidently, a convex body $K$ is of constant width if and only if $\triangle K$ is a ball. If $K$ is a centrally symmetric convex body in $\mathbb{R}^{n}$, then $h_{K}(u)=h_{-K}(u)=h_{K}(-u)$ implying that $w_{K}(u)=h_{K}(u)+h_{-K}(u)=2 h_{K}(u)$. So if the width function of a centrally symmetric convex body is constant, then its support function is also constant; hence the body must be a ball. Using the BrunnMinkowski inequality [see Theorem 7.1.1 in Schneider (2014)], we get that in the class of convex bodies of constant width $d$, the ball has the largest volume. In fact, the central symmetral of $K$ is $\triangle K=1 / 2(K+(-K))$ with the same width as $K$, but it is centrally symmetric; hence it is a ball with the same width function as $K$. On the other hand, the Brunn-Minkowski inequality gives that for the central symmetral (which is the ball of the same class of constant width)

$$
\operatorname{vol}_{n}(\triangle K)^{\frac{1}{n}}=\operatorname{vol}_{n}\left(\frac{1}{2}(K+(-K))\right)^{\frac{1}{n}} \geq \operatorname{vol}_{n}(K)^{\frac{1}{n}}
$$

as we stated. Now the question "Whose body has the minimal volume in a class of convex bodies of constant width $d$ ?" is natural. In the class of all planar convex sets of constant width $d$, the Reuleaux triangle has the least area. The first proofs of this theorem are contained in the papers by Lebesgue (1914) and by Blaschke (1915). Similar question was investigated by Pal (1921) who showed that the regular triangle has the least area among all convex sets of given thickness $d$. Recently, Campi, Colesanti and Gronchi investigated the minimum volume problem in Campi et al. (1996). They considered the 3-dimensional case and raised the following problems:

Problem 2 (Campi-Colesanti-Gronchi) Find a convex body of the minimum volume in each of the following classes:

(A) The class of convex bodies of constant width $d$;

(B) The class of convex bodies of thickness $d$.

Notice that the existence of solutions for each of these problems is guaranteed by standard compactness arguments. Problems A and B are still unsolved. On the other hand, several authors turned their attention to the classes of convex bodies involved in those problems [for a detailed history read the paper Campi et al. (1996)]. We consider only those bodies which are good candidates to solve these problems. First we mention here the Reuleaux tetrahedron which can be obtained from a regular tetrahedron with the edge of length $d$, as the intersection of four balls centred at the vertices of the tetrahedron with radius $d$. Unfortunately, Reuleaux tetrahedron is not a body of constant width: the midpoints of its two opposite curved edges have distance greater than $d$. Meissner and Schilling (1912) showed how to modify the Reuleaux tetrahedron to form a body of constant width by replacing three of its edge arcs by curved patches formed as the surfaces of rotation of a circular arc. Incidentally, as Meissner mentioned on p. 49 of Meissner (1911), the ball is the only body of constant width that is bounded only by spherical pieces. According to this, if three edge arcs are replaced (three that have a common vertex or three that form a triangle), two noncongruent shapes arising are called Meissner tetrahedra (see Figs. 2, 3, 4). 

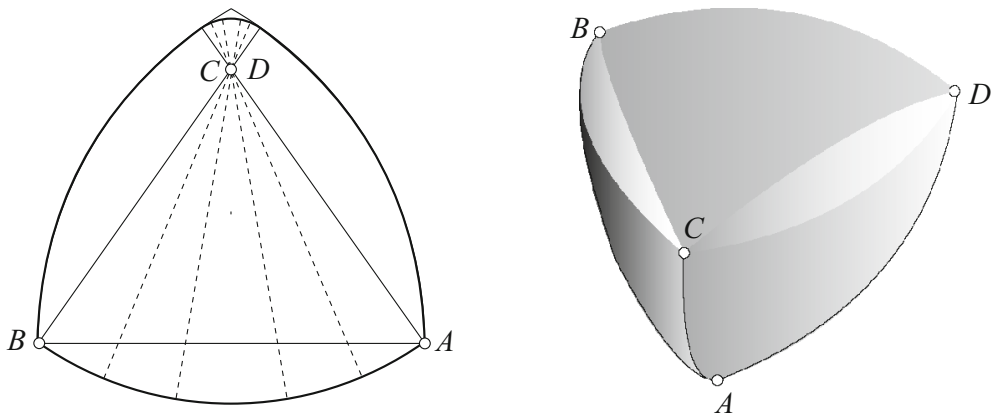

Fig. 2 The Meissner tetrahedron
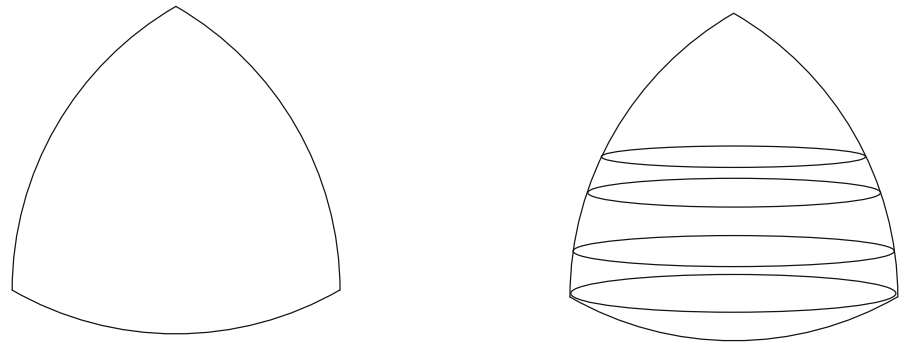

Fig. 3 The body of revolution with the minimal volume

Bonnesen and Fenchel conjectured in Bonnesen and Fenchel (1934):

Conjecture 1 (Bonnesen and Fenchel 1934) Meissner tetrahedra are the minimumvolume three-dimensional shapes of constant width.

This conjecture is still open. (I propose to read the nice paper of Kawohl and Weber (2011) on this conjecture.) In connection with this problem, Campi, Colesanti and Gronchi showed.

Theorem 2 (Campi et al. 1996) The minimum volume surface of revolution with constant width is the surface of revolution of a Reuleaux triangle through one of its symmetry axes.

A candidate to solve Problem B was proposed by Heil (1978); in this case the construction is based upon a tetrahedron, too. Namely, the Heil body is the convex hull of six circular arcs of radius $d$, centered at the mid-points of the edges of a regular tetrahedron with edges of length $d \sqrt{2}$, and the four vertices of a rescaled tetrahedron with edges of length $d(2 \sqrt{6}-\sqrt{2}) / 3$.

Conjecture 2 (Heil 1978) Heil body is the minimum-volume three-dimensional body in the class of convex bodies with given thickness.

Campi, Colesanti and Gronchi solved this problem for surfaces of revolution using the concept of a shaken body. Fix a plane $H$ orthogonal to $u \in \mathcal{S}^{n-1}$ and a closed 

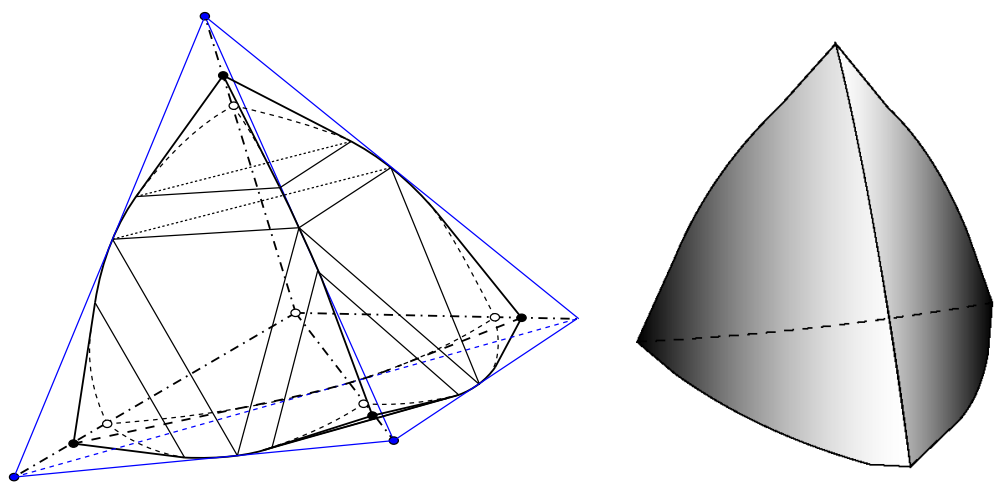

Fig. 4 The Heil body

half-space $H^{+}$bounded by $H$. The shaken body $S_{K}(u)$ of $K$ with respect to $u$ is the set contained in $H^{+}$such that for every line $l$ parallel to $u, S_{K}(u) \cap l$ is either a segment having an endpoint on $H$ and the same length as $K \cap l$, or the empty set, whether $l$ intersects $K$ or not [see Bonnesen and Fenchel (1934)]. Clearly, the shaken body is a convex body of the same volume as $K$. On the other hand, Campi at al. proved that the width function of the shaken body in the direction of the axis of a three-dimensional convex body of revolution is greater than the width function of $K$. From this the author showed

Theorem 3 (Campi et al. 1996) Among all three-dimensional convex bodies of revolution of a given thickness $d$, the unique body of the minimum volume is the cone generated by the revolution of a regular triangle with side $2 d / \sqrt{3}$ around one of its axes of symmetry.

\subsection{The Brightness Function, the Projection Body, and the Blaschke Body}

The brightness function of $K$ is also determined by the covariogram function. For a disk, it can be obtained by the rotation of the width function around the origin by $\pi / 2$. The brightness of $K$ in the direction of the unit vector $u$ is the $(n-1)$-dimensional volume of the orthogonal projection of $K$ to a hyperplane with the unit normal vector $u$. The brigthness function is the function

$$
b_{K}(u): u \mapsto \operatorname{vol}_{n-1}\left(K \mid u^{\perp}\right),
$$

where $\left(K \mid u^{\perp}\right)$ is the orthogonal projection of $K$ onto the hyperplane with the normal vector $u$. Our notes on the brigthness function of a disk is clear from Fig. 5. The covariogram function determines the brightness function of the body. In fact, for $u \in \mathcal{S}^{n-1}$,

$$
\lim _{r \mapsto 0+0} \frac{\mathrm{d}}{\mathrm{d} r} g_{K}(r u)=-\operatorname{vol}_{n-1}\left(K \mid u^{\perp}\right)=-b_{K}(u) .
$$




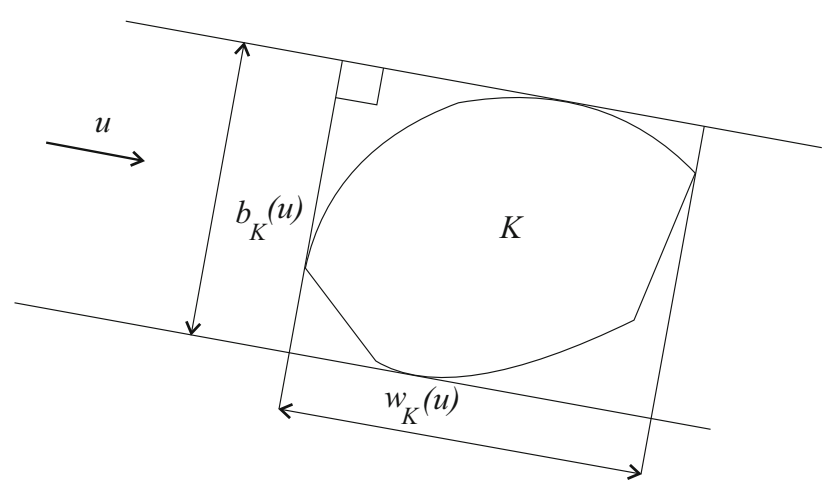

Fig. 5 The width function and the brightness function of a disk
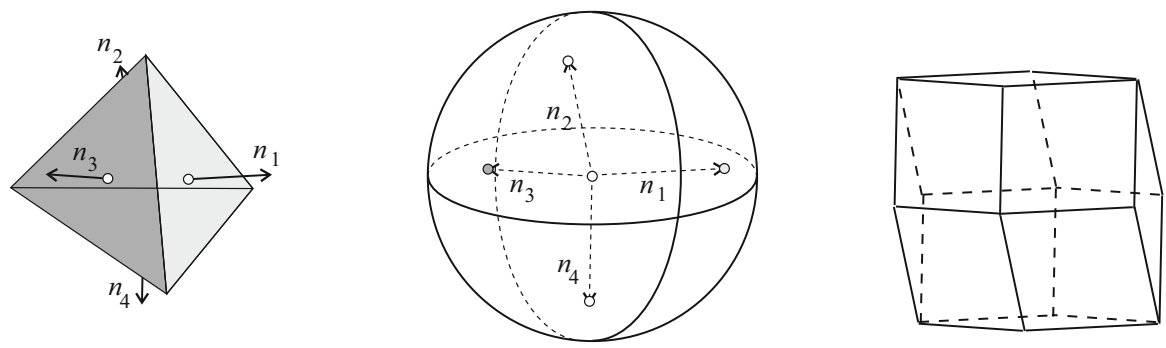

Fig. 6 The projection body of the regular tetrahedron

This follows from the facts that

$$
r \operatorname{vol}_{n-1}\left((K \cap(K+r u)) \mid u^{\perp}\right) \leq \operatorname{vol}_{n}(K \backslash(K+r u)) \leq r \operatorname{vol}_{n-1}\left(K \mid u^{\perp}\right),
$$

and $\lim _{r \mapsto 0+0} K \cap(K+r u)=K$. The answer for the question whether the brightness function determines or does not determine the body is known. Let us first introduce the projection body $\Pi K$ of $K$ as the uniquely defined body whose support function at the point $u$ is equal to the value of the brightness function of $K$ at $u$. If we restrict our investigations to centered convex bodies of $\mathbb{R}^{n}$, then from Alexandrov's projection theorem [see Theorem 3.3.6 in Gardner (1995)] we get that if $K_{1}$ and $K_{2}$ have the same brightness functions (or, equivalently, the projection bodies $\Pi K_{1}$ and $\Pi K_{2}$ agree), then $K_{2}$ is a translate of $K_{1}$. In general, as we will see later, there is a continuum of (generally non-congruent) sets with the same brightness function as $K$ with this property.

On Fig. 6, we can see the projection body of the regular tetrahedron. To determine the projection body of a polyhedron, we can use the Cauchy projection formula [see A.45 in Gardner (1995)], which relates the volumes of projections to the surface area measure as follows:

$$
h_{\Pi K}(u):=b_{K}(u)=\operatorname{vol}_{n-1}\left(K_{1} \mid u^{\perp}\right)=\frac{1}{2} \int_{\mathcal{S}^{n-1}}|\langle u, v\rangle| \mathrm{d} S_{n-1}(K, v) .
$$


The surface area measure of the regular tetrahedron is the measure concentrated to four vertices of a regular tetrahedron inscribed in the unit sphere. These points have equal measures (see $n_{i}$ on the figure). The integral then reduces to the sum of the four terms $\left|\left\langle u, n_{i}\right\rangle\right|, i=1, \ldots, 4$. Each term is the support function of a line segment $\left[0, \operatorname{vol}_{n-1}\left(F_{i}\right) n_{i}\right]$, where $F_{i}$ is the facet orthogonal to $n_{i}$. By the property of the support function, the projection body is the Minkowski sum of the four segments. Hence $\Pi K$ is a rhombic dodecahedron (see on the right of Fig. 6).

Let $K$ be a convex body of dimension $n$. The Gauss map $G$ sends a unit normal vector of the boundary of $K$ to the corresponding point of the sphere $\mathcal{S}^{n-1}$. We can define a measure on $\mathcal{S}^{n-1}$ by means of the Gauss map. For a set $H \subset \mathcal{S}^{n-1}$, we consider the set $G^{-1}(H)$ of those points of $K$, in which the unit normal vector is mapped by the Gauss map to a point of $H$. Let the measure of $H$ be the surface area of the set $G^{-1}(H)$. We denote by $S_{n-1}(K, \cdot)$ this surface area measure. Minkowki's existence theorem [see A.3.2. in Gardner (1995)] says that for a finite Borel measure on the unit sphere not concentrated on a great subsphere of $\mathcal{S}^{n-1}$ there is a convex body $K$, for which the surface area measure $S_{n-1}(K, \cdot)$ is the given one. Hence if $K$ is a convex body in $\mathbb{R}^{n}$, and $0 \leq t \leq 1$, then there is a unique convex body whose surface area measure is $(1-t) S_{n-1}(K, \cdot)+t S_{n-1}(-K, \cdot)$. The relation between the brightness function and the surface area measure of $K$ is the following: The condition that for all $u \in \mathcal{S}^{n-1}, \operatorname{vol}_{n-1}\left(K_{1} \mid u^{\perp}\right)=\operatorname{vol}_{n-1}\left(K_{2} \mid u^{\perp}\right)$ is equivalent to that for all $u \in \mathcal{S}^{n-1}, S_{n-1}\left(K_{1}, \cdot\right)+S_{n-1}\left(-K_{1}, \cdot\right)=S_{n-1}\left(K_{2}, \cdot\right)+S_{n-1}\left(-K_{2}, \cdot\right)$ [see Theorem 3.3.2 in Gardner (1995)]. This leads to another important observation: since for all $0 \leq t \leq 1$

$$
\begin{aligned}
& \left((1-t) S_{n-1}(K, \cdot)+t S_{n-1}(-K, \cdot)\right)+\left((1-t) S_{n-1}(-K, \cdot)+t S_{n-1}(K, \cdot)\right) \\
& \quad=S_{n-1}(K, \cdot)+S_{n-1}(-K, \cdot),
\end{aligned}
$$

the unique convex body, whose surface area measure is $(1-t) S_{n-1}(K, \cdot)+$ $t S_{n-1}(-K, \cdot)$, has the same brightness function as $K$. This also implies that their projection bodies are the same centred convex body.

The Blaschke body $\nabla K$ corresponds to $t=1 / 2$. The term projection class of $K$ is used for the class of all convex bodies $K^{\prime}$ for which $\Pi K=\Pi K^{\prime} . \nabla K$ is the unique body in a projection class with the largest volume [see Theorems 4.1.3 and 3.3.9 in Gardner (1995)]. From the proof [using Minkowski's first inequality, B.11 in Gardner (1995)], it follows that the volume of $K$ is equal to the volume of $\nabla K$ if and only if $K$ is centrally symmetric. This means that the only centrally symmetric element of a projection class is the Blaschke body. In Fig. 7, we drew the Blaschke body of the regular tetrahedron. The surface area measure of $-K$ is the reflected image of the surface area measure of $K$. Hence the surface area measure of the Blaschke body is concentrated to eight points with equal masses situated at the outward unit vectors of the facets of a regular octahedron; hence the body $\nabla K$ is the regular octahedron, which is the intersection of the regular tetrahedron $K$ and its reflected image $-K$ (here the origin is the common centroid of the two tetrahedra).

In dimension 3, a convex body has constant brightness if its shadow on any plane always has the same area. Among centrally symmetric convex bodies, the ball is the 

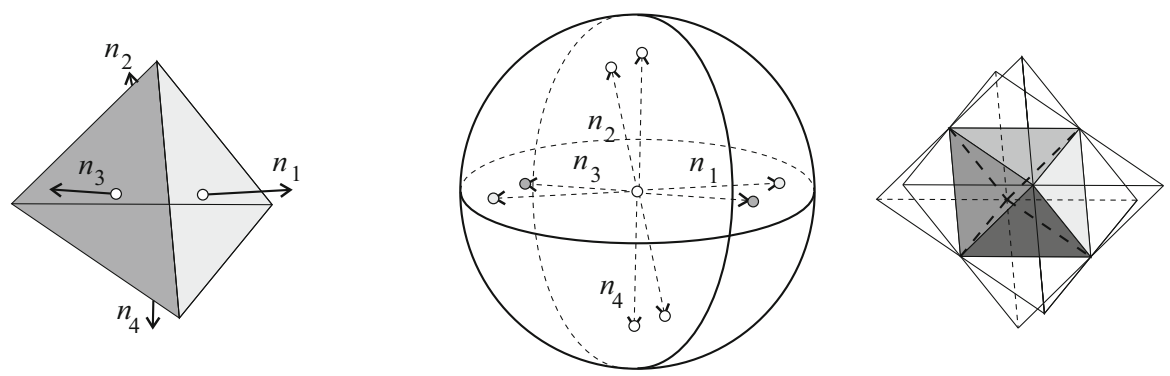

Fig. 7 The Blaschke body of the regular tetrahedron

Fig. 8 A nonspherical body of constant brightness

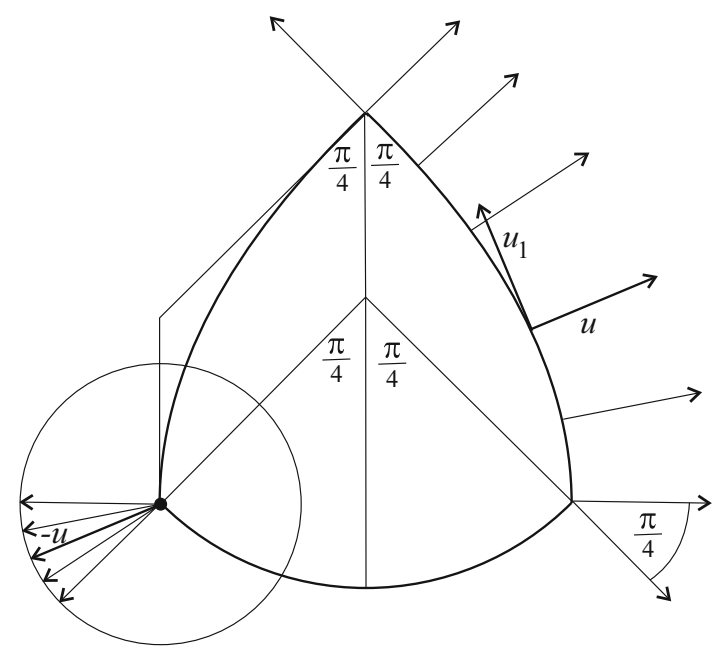

only one with constant brightness [Theorem 3.3.11 in Gardner (1995)]. Blaschke constructed the first example of a nonspherical convex body in $\mathbb{R}^{3}$ of constant brightness (see Blaschke 1949). He observed that such a body can be found in the class of solids of revolution with the $z$-axis as the axis of revolution. Let us briefly describe this nice construction. It is based on the fact that a convex body of class $C_{+}^{2}$ has a constant brightness if and only if the sum of the products of the principal radii at antipodal points is constant [see Theorem 3.3.14 in Gardner (1995)]. Blaschke described the 2-dimensional meridian section of the body $K$, which lies in the $\{x, z\}$-plane. Let $x_{u}$ be the point of this meridian, where the outer normal vector is $u$. The directions corresponding to the principal radii of Gauss curvature are $\left\{u_{1}, u_{2}\right\}$. We take $u_{1}$ in the plane of $\{u, z\}$, orthogonal to $u$ and $u_{2}$ orthogonal to the plane $\left\{u, u_{1}\right\}$. The second principal radius $R_{2}$ is the distance from $x_{u}$ to the $z$-axis measured along the line through $x_{u}$ parallel to $u$. We need

$$
R_{1}(u) R_{2}(u)+R_{1}(-u) R_{2}(-u)=\text { const }=c
$$

for all $u$. We can see the examined meridian in Fig. 8. Note that the base point of one of two opposite normal vectors is in the vertex of this meridian. Hence the smooth 
parts have to be of constant Gauss curvature with the same value (in a point of nonsmoothness, the Gauss curvature is infinity given that one of the principal radii is equal to zero). For a surface of revolution of the form

$$
F(x, \varphi)=(x \cos \varphi, x \sin \varphi, z(x)),
$$

the Gauss curvature $K$ is known to be

$$
\frac{1}{R_{1}(u) R_{2}(u)}=K=\frac{\grave{z} \ddot{z}}{x\left(1+\dot{z}^{2}\right)^{2}}
$$

By the substitution $t=\dot{z}^{2}, \dot{t}=2 \dddot{z} \ddot{z}$, we get from this in the case of $K \neq 0$ the differential equation

$$
\frac{\dot{t}}{(1+t)^{2}}=2 K x
$$

The general solution for $t$ is

$$
\dot{z}^{2}=t=-\frac{1+c+K x^{2}}{c+K x^{2}},
$$

where $c$ is an arbitrary constant. In our case of $K=1$, we also know the initial condition: $\dot{z}(0)=-1$. Hence $c=-1 / 2$, and

$$
z(x)= \pm \int_{0}^{x} \sqrt{\frac{1+2 x^{2}}{1-2 x^{2}}} \mathrm{~d} x+C
$$

with another constant $C$. Substituting $\cos v=\sqrt{2} x$ in this non-elementary integral we get

$$
z\left(\frac{\cos v}{\sqrt{2}}\right)=\mp \frac{1}{\sqrt{2}} \int_{\pi / 2}^{\cos ^{-1} \sqrt{2} x} \sqrt{1+\cos ^{2} v} \mathrm{~d} v+C= \pm \frac{1}{\sqrt{2}} \int_{\cos ^{-1} \sqrt{2} x}^{\pi / 2} \sqrt{2-\sin ^{2} v} \mathrm{~d} v+C
$$

The initial condition now is $z(\sqrt{2} / 2)=0$; hence

$$
C=\mp \frac{1}{\sqrt{2}} \int_{0}^{\pi / 2} \sqrt{2-\sin ^{2} v} \mathrm{~d} v
$$


Since $z(v) \geq 0$, and $\frac{1}{\sqrt{2}} \int_{0}^{\pi / 2} \sqrt{2-\sin ^{2} v} \mathrm{~d} v \geq \pm \frac{1}{\sqrt{2}} \int_{\cos ^{-1} \sqrt{2} x}^{\pi / 2} \sqrt{2-\sin ^{2} v} \mathrm{~d} v$, we have to choose the positive sign for $C$. Hence the only possibility is

$$
\begin{aligned}
z\left(\frac{\sqrt{2}}{2} \cos v\right) & =\frac{\sqrt{2}}{2}\left(\int_{0}^{\pi / 2} \sqrt{2-\sin ^{2} v} \mathrm{~d} v-\int_{\cos ^{-1} \sqrt{2} x}^{\pi / 2} \sqrt{2-\sin ^{2} v} \mathrm{~d} v\right) \\
& =\frac{\sqrt{2}}{2} \int_{0}^{\cos ^{-1} \sqrt{2} x} \sqrt{2-\sin ^{2} v} \mathrm{~d} v,
\end{aligned}
$$

which is the meridian proposed by Blaschke in Blaschke (1949).

A nice problem arose in the common investigation of width and brightness. In the plane, the two concepts are essentially the same and they give the same information on the body. In 3-space, this is not true; so we have the following question: Is every convex body in $\mathbb{R}^{3}$ of constant width and constant brightness a ball? The positive answer for $C^{2}$ bodies was given by Nakajima (1926). He showed that any convex body in $\mathbb{R}^{3}$ with constant width, constant brightness, and the boundary of class $C^{2}$ is a ball. In 2005 , Howard proved in Howard (2006) that the regularity assumption on the boundary is unnecessary, so balls are the only convex bodies of constant width and brightness.

Turning back to paper of Campi at al., we find the following problems:

Problem 3 (Campi-Colesanti-Gronchi) Find a convex body of the minimum volume in each of the following classes:

(C) The class of convex bodies of constant brightness $b$;

(D) The class of convex bodies of the minimal brightness $b$.

To get a partial solution of problem C), the authors proved the following

Theorem 4 (Campi et al. 1996) Let $K$ be a body of the minimum volume in the class of all convex bodies of constant brightness $b$. Then the surface area measure $S_{2}(K, \cdot)$ of $K$ has the following form:

$$
S_{2}(K, H)=\int_{H} s_{K}(z) \mathrm{d} z, \quad H \text { is a Borel set in } \mathcal{S}^{2},
$$

where $s_{K}(\cdot)$ is a non-negative function in $L_{1}\left(\mathcal{S}^{2}\right)$ such that

$$
\begin{aligned}
s_{K}(z)+s_{K}(-z) & =-\frac{2 b}{\pi} \text { almost ewerywhere in } \mathcal{S}^{2}, \\
s_{K}(z) s_{K}(-z) & =0 \text { almost ewerywhere in } \mathcal{S}^{2} .
\end{aligned}
$$

This theorem leads to further conjectures: 
Conjecture 3 (Campi-Colesanti-Gronchi on Problem C) Consider three pairwise orthogonal great circles on $\mathcal{S}^{2}$; they determine eight open regions. Define $s_{K}$ as a piecewise constant function whose values are 0 and $2 b / \pi$ on those regions alternately. Let $K$ be the unique convex body such that $s_{K}$ is the distribution of its surface area measure. Clearly, $K$ has constant brightness $b$, and the Gauss curvature is $\pi / 2 b$ at each regular point of $K$.

The authors conjectured that this body is a solution of Problem C.

Conjecture 4 (Campi-Colesanti-Gronchi on Problem $\mathrm{C}$ when the body is a body of revolution) The body constructed by Blaschke has the minimum volume among all convex bodies of revolution with constant brightness $b$.

To support this conjecture, they noticed that Blaschke's body of constant brightness can be constructed in the following manner: Fix on $\mathcal{S}^{2}$ a great circle as an equator and take the pair of parallel circles at a spherical distance $\pi / 4$ from it. In such a way $\mathcal{S}^{2}$ is divided into four open regions. Define $s_{K}$ to be a piecewise constant function whose values are 0 and $2 b / \pi$ on those regions alternately; $s_{K}$ is the distribution of the area measure of the convex body of revolution with constant brightness constructed by Blaschke (1949). In the nice paper Gronchi (1998), proved this conjecture:

Theorem 5 (Gronchi 1998) The n-dimensional Blaschke-Firey body is the unique (up to a rigid motion) element of the minimal volume in the class of all convex bodies of revolution with constant brightness $b$.

We note that problem D) is still open, although the regular simplex is a strong candidate to solve it.

\section{The Convex-Hull Function}

The convex-hull function of a convex body $K$ is the "dual" of the covariogram function. It also has been examined for a long time. The first paper related to it was written by Fáry and Rédei (1950). They proved that if one of the bodies moves on a line with constant velocity, then the volume of the convex hull is a convex function of time [see Satz.4 in Fáry and Rédei (1950)]. The general definition is the following:

Definition 1 Let $K$ be an $n$-dimensional convex compact body; associate with a translation vector $t \in \mathbb{R}^{n}$ the value $G_{K}(t)=\operatorname{vol} \operatorname{conv}\{K \cup(K+t)\}$. We call this function the convex-hull function, associated with the body $K$.

In dimension 2, this statement says that an intersection of the graph of the convexhull function with a plane parallel to the $z$-axis is a convex function. Later, several papers related to this observation suggested a lot of interesting problems [see the survey Horváth (2018) and the references therein]. It is interesting that the "basic problem" on the convex-hull function, whether the convex-hull function determines or does not determine the body $K$ was asked only recently by Á. Kurusa.

To answer this question, we have to collect some general information on the convexhull function. Obviously $G_{K}(0)=\operatorname{vol}(K)$, and its support is the whole $\mathbb{R}^{n}$. Denote 

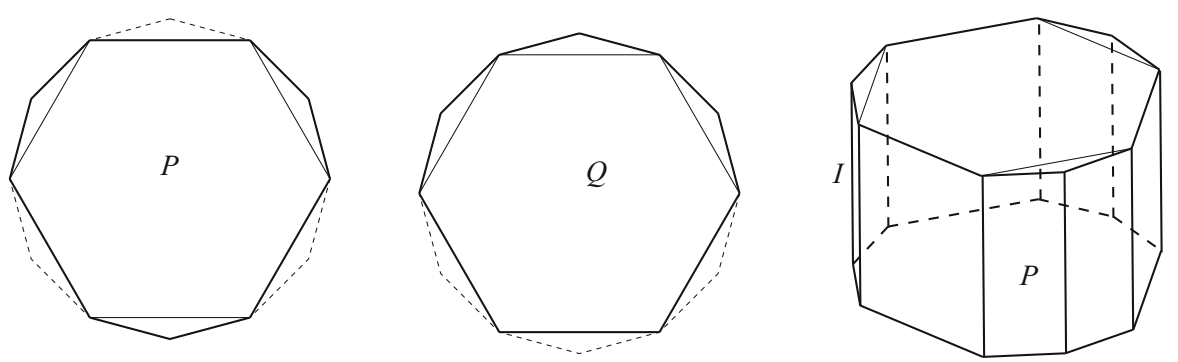

Fig. 9 Non-congruent nonagons with the same brightness function and with the same volume

by $u$ a unit vector and (as in previous sections) by $K \mid u^{\perp}$ the orthogonal projection of $K$ onto a hyperplane with the normal vector $u$.

Lemma 1 Let $\alpha$ be an arbitrary real number, and $u \in \mathcal{S}^{n-1}$ be a unit vector in $\mathbb{R}^{n}$. Then we have

$$
G_{K}(\alpha u)=\operatorname{vol}_{n}(K)+|\alpha| \operatorname{vol}_{n-1}\left(K \mid u^{\perp}\right)
$$

consequently, the function $u \mapsto G_{K}(u)-\operatorname{vol}_{n}(K)$ is the brightness function of $K$. Conversely, the brightness function of $K$ and the value $\operatorname{vol}_{n} K$ determine the convexhull function of $K$.

Proof The equality (12) is an easy consequence of the Cavalieri principle [see also Sect. A.5 in Gardner (1995)]. The second statement follows from the definition of the brightness function. The third statement is an immediate consequence of the equality (12).

Remark In the case when the brightness function of $K$ determines the body $K$, it also determines the convex-hull function of $K$. On the other hand, we discussed earlier that there are several bodies with the same brightness function. The only question is that if the volume and the brightness function are both known, then whether the body is determined or not. Since the covariogram function also has these two properties in dimension $n$ for $n \geq 4$, the answer should be "no". We know that on the plane there are two non-congruent convex polygons with the same width function and the same area. To get an example, take two congruent regular hexagons and add (in a suitable manner) to each of them three congruent suitable isosceles triangles; we will get two nonagons, which will not be congruent to each other. (In Fig. 9 we can see the two nonagons $P$ and $Q$, which are non-congruent parts of a regular dodecagon.) We might observe that the support functions $h_{P}$ and $h_{Q}$ of $P$ and $Q$ have the property that $\left\{h_{P}(u), h_{P}(-u)\right\}=$ $\left\{h_{Q}(u), h_{Q}(-u)\right\}$. Hence $w_{P}(u)=w_{Q}(u)$ for all $u$; furthermore, the width function is a rotated copy of the brightness function; thus this example has the two required properties.

Using these nonagons $P$ and $Q$, we can construct a 3-dimensional pair of polyhedra with the same brightness function and volumes. Consider the prisms $H_{P}:=P \times I$ and $H_{Q}:=Q \times I$, where $I$ is a segment orthogonal to the common plane $H$ of $P$ and $Q$. By the following theorem, these prisms have the required properties. 
Theorem 6 Let $P$ and $Q$ be convex bodies of dimension $n$ with the same brightness function and volume. Let I be a segment orthogonal to that subspace $H$ of the $(n+1)$ dimensional Euclidean space, which contains $P$ and $Q$. Then the prisms $H_{P}$ and $H_{Q}$ also have the same brightness function and $(n+1)$-dimensional volume.

Proof The equality $\operatorname{vol}_{n+1}\left(H_{P}\right)=\operatorname{vol}_{n+1}\left(H_{Q}\right)$ is obvious.

First we prove that the orthogonal projections $P^{\prime}$ and $Q^{\prime}$ of $P$ and $Q$ to a subspace $\Pi$ with the unit normal vector $u$ have the same Hausdorff measure. (In the case when $\Pi$ is not orthogonal to $H$, the projection has a non-zero $n$-dimensional volume, which agrees with the measure above, and in the orthogonal case this measure is equal to the $(n-1)$-dimensional volume of the projection.) Let the unit normal vector of $H$ be $h$ and $I=\alpha h$. Since $P$ and $Q$ have the same $n$-dimensional volume, we have $\operatorname{vol}_{n}\left(P^{\prime}\right)=|\langle u, h\rangle| \operatorname{vol}_{n}(P)=|\langle u, h\rangle| \operatorname{vol}_{n}(Q)=\operatorname{vol}_{n}\left(Q^{\prime}\right)$. If it is non-zero, then the projections have the same $n$-dimensional volume. If it is zero, $H$ and $\Pi$ are orthogonal to each other, and the projections of $P^{\prime}$ and $Q^{\prime}$ are the respective shadows of $P$ and $Q$ on an $(n-1)$-dimensional subspace of $H$. Since their brightness functions agree, the $(n-1)$-dimensional volumes of $P^{\prime}$ and $Q^{\prime}$ are equal to each other, as we stated.

Assume that $H$ and $\Pi$ are not orthogonal. Then the projection $\left(H_{P} \mid u^{\perp}\right)$ is the convex hull of the projections $P^{\prime}$ and $P^{\prime}+(\alpha h-\langle u, \alpha h\rangle u)$ of the polytopes $P$ and $P+\alpha h$ of dimension $n$, where + denotes the vector sum. Hence by Eq. (12)

$$
\operatorname{vol}_{n}\left(H_{P} \mid u^{\perp}\right)=\operatorname{vol}_{n}\left(P^{\prime}\right)+|\alpha||(h-\langle u, h\rangle u)| \operatorname{vol}_{n-1}\left(P^{\prime} \mid(h-\langle u, h\rangle u)^{\perp}\right) .
$$

On the other hand, we have

$$
\left(P^{\prime} \mid(h-\langle u, h\rangle u)^{\perp}\right)=\left((P \mid \Pi) \mid(h-\langle u, h\rangle u)^{\perp}\right)=(P \mid H \cap \Pi),
$$

since $\langle v, u\rangle=0$ and $\langle v, h-\langle u, h\rangle u\rangle=0$ ensures $\langle v, h\rangle=0$. Thus

$$
\begin{aligned}
\operatorname{vol}_{n}\left(H_{P} \mid u^{\perp}\right) & =\operatorname{vol}_{n}\left(P^{\prime}\right)+|\alpha||(h-\langle u, h\rangle u)| \operatorname{vol}_{n-1}(P \mid H \cap \Pi) \\
& =\operatorname{vol}_{n}\left(Q^{\prime}\right)+|\alpha||(h-\langle u, h\rangle u)| \operatorname{vol}_{n-1}(Q \mid H \cap \Pi)=\operatorname{vol}_{n}\left(H_{Q} \mid u^{\perp}\right),
\end{aligned}
$$

as we stated.

If $H$ and $\Pi$ are orthogonal, then

$$
\operatorname{vol}_{n}\left(H_{P} \mid u^{\perp}\right)=\operatorname{vol}_{n-1}(P \mid H \cap \Pi)=\operatorname{vol}_{n-1}(Q \mid H \cap \Pi)=\operatorname{vol}_{n}\left(H_{Q} \mid u^{\perp}\right)
$$

immediately gives the required result.

Using Theorem 6 we get an example in arbitrary dimension for non-congruent convex bodies with the same brightness function and volume.

The following problem leads to some interesting relations between volume functions.

Definition 2 (Horváth and Lángi 2014) If for a convex body $K \subset \mathbb{R}^{n}$, $\operatorname{vol}_{n}(\operatorname{conv}(K \cup$ $(v+K)))$ has the same value for any non-overlapping translate $v+K$ of $K$, which has a common point with $K$, then we say that $K$ satisfies the translative constant volume property. 
If $K$ is centered and satisfies the translative constant volume property, then $G_{K}(x)$ depends only on the norm $\|x\|_{K}$ of $x$. In fact, if $K$ is centered then it defines a norm $\|\cdot\|_{K}$, and $v+K$ touches $K$ if and only if $\|v\|_{K}=2$. Let $v=\alpha u$; then $\|v\|_{K}=|\alpha|\|u\|_{K}$ where $u$ is a Euclidean unit vector. From the translative constant volume property, it follows that

$$
\begin{aligned}
\text { const. } & =G_{K}(\alpha u)=\operatorname{vol}_{n}(K)+|\alpha| \operatorname{vol}_{n-1}\left(K \mid u^{\perp}\right) \\
& =\operatorname{vol}_{n}(K)+\frac{\|v\|_{K}}{\|u\|_{K}} \operatorname{vol}_{n-1}\left(K \mid u^{\perp}\right)=\operatorname{vol}_{n}(K)+2 \frac{\operatorname{vol}_{n-1}\left(K \mid u^{\perp}\right)}{\|u\|_{K}}
\end{aligned}
$$

This implies that $\frac{\operatorname{vol}_{n-1}\left(K \mid u^{\perp}\right)}{\|u\|_{K}}$ is constant for Euclidean unit vectors, and hence the value of $G_{K}(\alpha u)$ depends only on the value of $\|\alpha u\|_{K}$, as we have claimed. Hence the question which bodies satisfy the translative constant volume property is analogous to the question of Meyer et al. (1993) on covariogram stated in 1993. They proved that if $K$ is a centred body such that $g_{K}(x)$ depends only on the norm $\|x\|_{K}$ of $x$, then $K$ is an ellipsoid.

We recall that a 2-dimensional $o$-symmetric convex curve is a Radon curve if, for the convex hull $K$ of a suitable affine image of the curve, it is true that $K^{\circ}$ is a rotated copy of $K$ by $\frac{\pi}{2}$ (cf. Martini and Swanepoel 2006). The concept of a Radon curve was also raised in connection with the concept of Birkhoff orthogonality. Radon gave the first example in a Minkowski plane, in which the Birkhoff orthogonality relation is a symmetric one. On the other hand, it can be proved that, in a higher dimension, the Birkhoff orthogonality is a symmetric relation if and only if the norm is Euclidean, e.g. the unit ball of the space is an ellipsoid. The long history of this nice concept can be found in Sect. 4.7 of the book Thompson (1996). We also suggest to read the original work of Radon (1916). In the paper Horváth and Lángi (2014), we can find the following theorem:

Theorem 7 (Horváth and Lángi 2014) For any disk (planar convex body) K, the following properties are equivalent.

(1) $K$ satisfies the translative constant volume property.

(2) The boundary of the central symmetral of $K$ is a Radon curve.

(3) $K$ is a body of constant width in the Radon norm.

This statement motivates a new conjecture, since it is known [cf. Alonso and Benítez (1989) or Martini and Swanepoel (2006)] that for $d \geq 3$, if every planar section of a normed space is Radon, then the space is Euclidean; that is, its unit ball is an ellipsoid.

Conjecture 5 (Horváth and Lángi 2014) Let $d \geq 3$. If some centrally symmetric convex body $K \subset \mathbb{R}^{d}$ satisfies the translative constant volume property, then $K$ is an ellipsoid.

If $K$ and $K+t_{p}$ are touching at the point $p \in K$, then $p-t_{p} \in K$ and from Eq. (12) we get that $G_{K}\left(t_{p}\right)=\operatorname{vol}(K)+\left|t_{p}\right| \operatorname{vol}_{n-1}\left(K \mid t_{p}^{\perp}\right)$. The chord $\left[p, p-t_{p}\right]$ of $K$ is a so-called affine diameter of $K$. The general properties of affine diameters are described in the survey of Soltan (2005). In the centrally symmetric case, we know that a chord containing the center is an affine diameter. These affine diameters determine 
the radial function of the body $K$. If the origin $x$ is the center of $K$, then we define the radial function by the equality:

$$
\rho_{K}(u):=\sup \{t \in \mathbb{R}: t u \in K\} .
$$

The radial function can be considered for all convex bodies such that the origin is an interior point of $K$. There is the following relation between the support function and the radial function:

$$
\rho_{K}(u)=\frac{1}{h_{K^{\circ}}(u)},
$$

where $K^{\circ}$ is the polar body of $K$. The polar projection body of $K$ is the body $\Pi^{\circ} K:=$ $\Pi K^{\circ}$. An old problem is the so-called polar projection problem; it was raised by Petty (1971) and is mentioned by Gruber (1987) and Lutwak in Lutwak (1993).

Conjecture 6 (Petty 1971) If some centrally symmetric convex body $K \in \mathbb{R}^{d}$ satisfies $\Pi^{\circ} K=\lambda K$, then $K$ is an ellipsoid.

This problem has a form also in the non-symmetric case, asking which bodies have the property that their projection bodies and difference bodies are polars of each other. Martini (1991) proved that the only polytope with this property is the simplex. The reason why we mentioned here the polar projection problem is that it is equivalent to the conjecture on translative constant volume property. (We will prove it in a forthcoming paper.)

\section{Remarks on the Homothetic Versions of the Above Problems}

First of all, I would like to mention the paper of Meyer et al. (1993) in which we can find the following theorem:

Theorem 8 (Martini 1991) Let $K \subset \mathbb{R}^{n}$ be a centrally symmetric convex body. If for some $\tau$ the volume $\operatorname{vol}_{n}(K \cap\{\tau K+x\})$ depends only on the Minkowski norm $\|x\|_{K}$, then $K$ is an ellipsoid.

For the convex-hull function, an analogous result was proven by Castro (2015). He proved the following two statements:

Theorem 9 (Castro 2015) Let $K \subset \mathbb{R}^{n}$ be a convex body with the origin o in its interior. If there is a number $\lambda \in(0,1)$ such that $\operatorname{vol}_{n} \operatorname{conv}\{K \cup(\lambda K+x)\}$ depends only on the Euclidean norm $\|x\|$, then $K$ is a Euclidean ball.

Theorem 10 (Castro 2015) Let $K \subset \mathbb{R}^{n}$ be a convex body with the origin o in its interior, and let $L \subset \mathbb{R}^{n}$ be a centrally symmetric convex body centered at the origin. If there is a number $\lambda \in(0,1)$ such that $\operatorname{vol}_{n} \operatorname{conv}\{K \cup(\lambda K+x)\}$ depends only on the Minkowski norm $\|x\|_{L}$, then $L$ is homothetic to $K$. 
Unfortunately, the witty proofs of these statements cannot be applied to the case of Conjecture 5. However, these results suggest the following problem.

Definition 3 Denote by

$$
G_{K, \lambda}(t):=\operatorname{vol}_{n} \operatorname{conv}\{K \cup(\lambda K+t)\}
$$

the $\lambda$-homothetic convex-hull function of $K$.

Problem 4 Does the $\lambda$-homothetic convex-hull function $G_{K, \lambda}(t)$ determine the body $K$ ? What can we say about $K$ if we know more $\lambda$-homothetic convex-hull functions of it?

Acknowledgements Open access funding provided by Budapest University of Technology and Economics (BME).

Open Access This article is licensed under a Creative Commons Attribution 4.0 International License, which permits use, sharing, adaptation, distribution and reproduction in any medium or format, as long as you give appropriate credit to the original author(s) and the source, provide a link to the Creative Commons licence, and indicate if changes were made. The images or other third party material in this article are included in the article's Creative Commons licence, unless indicated otherwise in a credit line to the material. If material is not included in the article's Creative Commons licence and your intended use is not permitted by statutory regulation or exceeds the permitted use, you will need to obtain permission directly from the copyright holder. To view a copy of this licence, visit http://creativecommons.org/licenses/by/4.0/.

\section{References}

Alonso, J., Benítez, C.: Orthogonality in normed linear spaces: a survey. II. Relations between main orthogonalities. Extracta Math. 4, 121-131 (1989)

Averkov, G., Bianchi, G.: Retrieving convex bodies from restricted covariogram functions. Adv. Appl. Probab. 39, 613-629 (2007)

Bianchi, G., Segala, F., Volcic, A.: The solution of the covariogram problem for plane $C_{2}^{+}$- bodies. J. Differ. Geom. 60, 177-198 (2002)

Bianchi, G.: Matheron's conjecture for the covariogram problem. J. Lond. Math. Soc. (2) 71, 203-220 (2005)

Bianchi, G.: The covariogram determines three-dimensional convex polytopes. Adv. Math. 220, 1771-1808 (2009)

Blaschke, W.: Konvexe Bereiche gegebener konstanter Breite und kleinsten Inhalts. Math. Ann. 76(4), 504-513 (1915)

Blaschke, W.: Kreis and Kugel. Chelsea, New-York (1949)

Bonnesen, T., Fenchel, W.: Theorie der konvexen Körper. Springer, Berlin (1934)

Campi, S., Colesanti, A., Gronchi, P.: Minimum problems for volume of convex bodies. In: Marcellini, Talenti, Vesentini (eds) Partial differential equations and applications, Collected papers in Honour of Carlo Pucci. Marcel Decker (1996)

Castro, J.J.: The volume of the convex hull of a body and its homothetic copies. Am. Math. Mon. 122(5), 486-489 (2015)

Croft, H.T., Falconer, K.J., Guy, R.K.: Unsolved Problems in Geometry, vol. 2. Springer, New York (1991)

Fáry, I., Rédei, L.Der: zentralsymmetrische Kern und die zentralsymmetrische Hülle von konvexen Körpern. Math. Ann. 122, 205-220 (1950)

Gardner, R.J.: Geometric Tomography. Cambridge University Press, Cambridge (1995)

G.Horváth, Á.: Volume of convex hull of two bodies and related problems. In: Marston C, Antoine D, Ivic WA (eds) (szerk.) Discrete Geometry and Symmetry. Springer Proceedings in Mathematics and Statistics; 234. (2018) ISBN:978-3-319-78433-5 
G.Horváth, Á., Lángi, ZS.: On the volume of the convex hull of two convex bodies. Monatshefte für Mathematik 174/2, 219-229 (2014)

Goodey, P., Schneider, R., Weil, W.: On the determination of convex bodies by projection functions. Bull. Lond. Math. Soc. 29, 82-88 (1997)

Gronchi, P.: Bodies of constant brightness. Arch. Math. 70(6), 489-498 (1998)

Gruber, P.M.: Radons contributions to convexity. In: Johan Radon Collected Works, Vol. 1, Birkhauser, Basel, pp 331-342 (1987)

Heil, E.: Kleinste konvexe Korper gegebener Dicke. Preprint no. 453, Technische Hochschule Darmstadt, (1978)

Howard, R.: Convex bodies of constant width and constant brightness. Adv. Math. 204, 241-261 (2006)

Kawohl, B., Weber, C.: Meissner's mysterious bodies. Math. Intell. 33(3), 94-101 (2011)

Lebesgue, H.: Sur le probleme des isoperimetres et sur le domainesde largeur constante. Bull. Soc. Math. Franc, C.R. 72-76 (1914)

Lutwak, E.: Selected affine isoperimetric inequalities. In: Gruber, P.M., Wills, J. (eds.) Handbook of Convex Geometry, Vol. A. North-Holland, pp 151-176 (1993)

Martini, H.: Convex polytopes whose projection bodies and difference sets are polars. Discrete Comput. Geom. 6, 83-91 (1991)

Martini, H., Swanepoel, K.J.: Antinorms and radon curves. Aequ. Math. 71, 110-138 (2006)

Matheron, G.: Le covariogramme géometrique des compacts convexes de $\mathbb{R}^{2}$, Technical report 2/86, 54 . Centre de Géostatistique, Ecole des Mines de Paris (1986)

Meissner, E.: Über Punktmengen konstanter Breite, Vierteljahrsschr. Nat.forsch. Ges. Zür.,56, 42-50 (1911). http://www.archive.org/stream/vierteljahrsschr56natu\#page/n53/mode/2up

Meissner, E., Schilling, Fr: Drei Gipsmodelle von Flachen konstanter Breite. Z. Math. Phys. 60, 92-92 (1912)

Meyer, M., Reisner, S., Schmuckenschläger, M.: The volume of the intersection of a convex body with its translates. Mathematika 40, 278-289 (1993)

Nagel, W.: Orientation-dependent chord length distributions characterize convex polygons. J. Appl. Probab. 30, 730-736 (1993)

Nakajima, S.: Eine charakteristicische Eigenschaft der Kugel. Jber. Deutsche Math.-Verein 35, 298-300 (1926)

Pal, J.: Ein Minimumproblem fur Ovale. Math. Ann. 83, 311-320 (1921)

Petty, C.M.: Isoperimetric problems. In: Proc. Conf. Convexity and Combinatorial Geometry, Univ. Oklahoma. University Oklahoma, pp 26-41 (1971)

Radon, J.: Über eine besondere Art ebener konvexer Kurven. Bel Sachs Akad Wiss Leipzig 68, 131-134 (1916)

Schmitt, M.: On two inverse problems in mathematical morphology. In: Mathematical Morphology in Image Processing, Dekker, New York, pp 151-169 (1993)

Schneider, R.: Convex Bodies: The Brunn-Minkowski theory, second expanded edition. In: Encyclopedia of Mathematics and its Applications 151. Cambridge University Press, Cambridge (2014)

Soltan, V.: Affine diameters of convex bodies-a survey. Expo. Math. 23, 47-63 (2005)

Thompson, A.C.: Minkowski Geometry, Encyclopedia of Mathematics and its Applications 63. Cambridge University Press, Cambridge (1996)

Publisher's Note Springer Nature remains neutral with regard to jurisdictional claims in published maps and institutional affiliations. 